\title{
Research Progress on Mechanism of Neuroprotective Roles of Apelin-13 in Prevention and Treatment of Alzheimer's Disease
}

\author{
Teng Wan ${ }^{1,2} \cdot$ Mingyuan $\mathrm{Fu}^{2} \cdot$ Yan Jiang ${ }^{4} \cdot$ Weiwei Jiang ${ }^{2} \cdot$ Peiling $\mathrm{Li}^{2} \cdot$ Shouhong $\mathrm{Zhou}^{1,3}$
}

Received: 7 July 2021 / Revised: 2 September 2021 / Accepted: 3 September 2021 / Published online: 13 September 2021

(c) The Author(s), under exclusive licence to Springer Science+Business Media, LLC, part of Springer Nature 2021

\begin{abstract}
Alzheimer's disease (AD) is the most common type of dementia. Currently, more than 50 million people live with dementia worldwide, and this number is expected to increase. Some of the typical pathological changes of AD include amyloid plaque, hyperphosphorylation of tau protein, secretion of inflammatory mediators, and neuronal apoptosis. Apelin is a neuroprotective peptide that is widely expressed in the body. Among members of apelin family, apelin-13 is the most abundant with a high neuroprotective function. Apelin-13/angiotensin domain type 1 receptor-associated proteins (APJ) system regulates several physiological and pathophysiological cell activities, such as apoptosis, autophagy, synaptic plasticity, and neuroinflammation. It has also been shown to prevent $\mathrm{AD}$ development. This article reviews the research progress on the relationship between apelin-13 and $\mathrm{AD}$ to provide new ideas for prevention and treatment of $\mathrm{AD}$.
\end{abstract}

Keywords Apelin-13 $\cdot$ AD $\cdot$ Amyloid beta $\cdot$ Neuroprotection

\section{Introduction}

Alzheimer's disease (AD) is a progressive neurodegenerative disease which is considered the most common cause of dementia [1]. The recent diagnostic criteria and guidelines for $\mathrm{AD}$ indicate that brain changes in patients with $\mathrm{AD}$ begin before symptoms such as memory loss. Therefore, intervention for early brain changes is critical in preventing and decelerating development of the disease [2]. Most patients in an advanced stage of the disease need nonstop care, which brings great pressure on their family and society

Teng Wan, Mingyuan Fu and Yan Jiang have contributed equally to the work and should be considered co-first authors.

Shouhong Zhou

112019023@glmc.edu.cn; 723285991@qq.com

1 Guangxi Key Laboratory of Brain and Cognitive Neuroscience, Guilin Medical University, Guilin 541199, Guangxi, China

2 Department of Physiology, Hengyang Medical College, University of South China, Hengyang 421001, Hunan, China

3 Department of Physiology, Basic Medical College, Guilin 541199, Guangxi, China

4 Department of Epidemiology and Health Statistics, School of Public Health, Xiangnan University, Chenzhou 423043, China at large. Such problems have especially become more apparent during COVID-19 [3]. Apelin-13 is acknowledged to be the predominant neuropeptide in neuroprotection functioning in preventing the onset of $\mathrm{AD}$ and arresting $\mathrm{AD}$ 's progression [4]. First, apelin-13 exerts its neuroprotective effects by acting directly or indirectly on anti-apoptosis, autophagy regulation, promoting microglial polarization and improving neuronal synaptic plasticity, which help in arresting the progression of $\mathrm{AD}$ [5]. Second, it reduces the production of $\beta$-amyloid protein $(A \beta)$ and phosphorylation of tau protein and then lowers the risk of AD occurrence. In addition, apelin-13 arrests the progression of $\mathrm{AD}$ through reducing oxidative stress, anti-neuroinflammation and other mechanisms [6]. This study reviews the research progress on the regulatory role of apelin-13 in cell activity and neuronal microenvironment associated with $\mathrm{AD}$.

\section{Alzheimer's Disease}

Globally, there are over 50 million patients with dementia with most of them having Alzheimer's disease. With the aging trend of the world population, the number of patients with $\mathrm{AD}$ in the world is expected to double by the year 2050 [1]. The main pathological hallmarks of $A D$ are amyloid plaques. They consist of aggregated $A \beta$, neurofibrillary 
tangles (NFTs) contributed by hyperphosphorylation of tau protein in general neurons [7]. Aggregation of $A \beta$ causes a decrease in synapse number and a reduction of synaptic plasticity because of persistent neuronal damage in the brain. Neurofibrillary tangles (NFTs) also perturb the transport of nutrients and essential molecules in neurons, leading to neuron death [8]. The brains of patient with late-stage AD manifest inflammation, significant atrophy and widespread fragments of dead neurons [9]. Currently, the amyloid hypothesis is generally adopted [10]. However, recent research shows that $\mathrm{A} \beta$ is proposed to regulate learning and memory, angiogenesis, neurogenesis, repair leaks in the blood-brain barrier (BBB) and act as an antimicrobial peptide and tumor suppressor $[11,12]$. It has been reported that many roles of $A \beta$ contradicts the traditional concept. This provides a novel insight into role of $\mathrm{A} \beta$ in physiological regulation. However, these outcomes are affected by multiple variables and hence statistical research as well as further experimental research are required to account for the heterogeneity and the peculiar phenomena.

Although the effect of anti-A $\beta$ therapy on blocking $\mathrm{AD}$ progression is striking, it's not the only target for AD therapy [13]. Another typical pathological characteristic of AD is the formation of NFTs [14]. In addition, neuroinflammatory reactions driven by microglia and astrocytes also play a prominent role in the pathogenesis of AD [9]. A series of cellular activities including autophagy, apoptosis, lipid metabolism and synaptic plasticity also indicates the pathogenesis of $\mathrm{AD}$ [15]. The pathogenesis of $\mathrm{AD}$ is orchestrated through plenty of regulation mechanisms [16, 17]. Interactive networks bring difficulties to further research and simultaneously provide more targets and options for AD's treatment.

\section{Overview of Apelin-13}

Apelin is a signaling molecule for impulse transmission between neurons [18]. It is widely expressed in many tissues and organs containing central nervous system (CNS), kidney and other peripheral organs [19]. The plasma content of apelin-13 is the highest and it is the main neuroprotective peptide. As the endogenous ligand of angiotensin domain type 1 receptor-associated proteins (APJ), apelin-13 system participates in several physiological and pathological processes such as vasculopathy, energy metabolism and maintenance of humoral homeostasis [20, 21]. Apelin-13/APJ system promotes proliferation of endothelial cells (ECs), which is a pivotal component of BBB [22]. Furthermore, apelin-13 plays a prominent role in repair of myocardial infarction [23]. Expression of apelin-13/APJ is down-regulated with aging. Knockout of apelin-13 and APJ gene accelerates aging in mouse model, while restoration of apelin-13 gene restore vitality, behavior recovery and circadian rhythm phenotype [24]. It has been reported that the muscle function of mice lacking apelin-13 or its receptors deteriorates significantly with aging [25]. Elsewhere, experimental studies have indicated that apelin-13 leads to neuroprotection. This may be partly related to inhibition of autophagy and regulation of apoptosis in neurodegenerative disease [26]. Apelin-13 acts as an anti-inflammation factor by exerting an inhibitory effect on inflammation [27]. Additionally, the apelin-13/ APJ system alleviates acute lung injury or acute respiratory distress syndrome by repressing oxidative reaction induced by mitochondrial reactive oxygen species (ROS), mitochondrial apoptosis and inflammatory response [6]. Furthermore, apelin-13 improves insulin sensitivity, lower blood glucose and blood lipids hence regulates the pathology of tumor [28-31]. It has protective effects in some neurodegenerative situations. A previous study suggests that apelin- 13 plays a protective role against pentylenetetrazole (PTZ)-induced toxicity by calcium blocking, antioxidant, anti-inflammatory and anti-apoptotic activity [32]. Roles of apelin-13 in antiaging, anti-inflammation, anti-oxidation and proliferation as well as repair-promotion, are found firmly associated with AD. Therefore apelin-13 has shown to be a neuroprotective factor, which may be a suppressor for pathogenesis and development of AD (Fig. 1).

\section{Mechanism in Inhibition Effects of Apelin-13 on AD}

\section{Alzheimer's Disease, Apelin-13 and Apoptosis Inhibition}

\section{Alzheimer's Disease and Apoptosis}

Abnormal apoptosis of non-regenerative neurons in CNS contribute to a structural and functional damage of neural network. This has been demonstrated to be closely correlated with multiple neurodegenerative diseases [33]. It has been reported that neuronal apoptosis induced by $\mathrm{A} \beta$, amyloid precursor protein (APP), ROS and other injurious factors causes pathogenesis of $\mathrm{AD}$ [34]. $\mathrm{A} \beta$ has been shown to induce nuclear translocation by regulating Yes-related proteins (YAP) and then promote expression and activation of Bcl-2-associated X protein (Bax), which eventually cause neuronal apoptosis [35]. Ischemic stroke-conditions that affect the brain and its blood supply is a common risk factor of AD [36]. Research studies have indicated that endoplasmic reticulum stress (ERS) and subsequently triggered unfolded protein response may be a crucial role in ischemia/ reperfusion (I/R)-induced neuronal apoptosis in ischemic stroke [37]. 
Fig. 1 Overview of the main effects of Apelin-13 on Alzheimer's disease. Apelin-13 exerts a positive effect on neurodegeneration by promoting improvement of synaptic plasticity, blood brain barrier (BBB) integrity, Apolipoprotein E4 (ApoE4) internalization and inhibiting neuroinflammation, apoptosis, autophagy, oxidative stress, angiotensin signaling and cerebrovascular amyloid angiopathy (CAA) formation

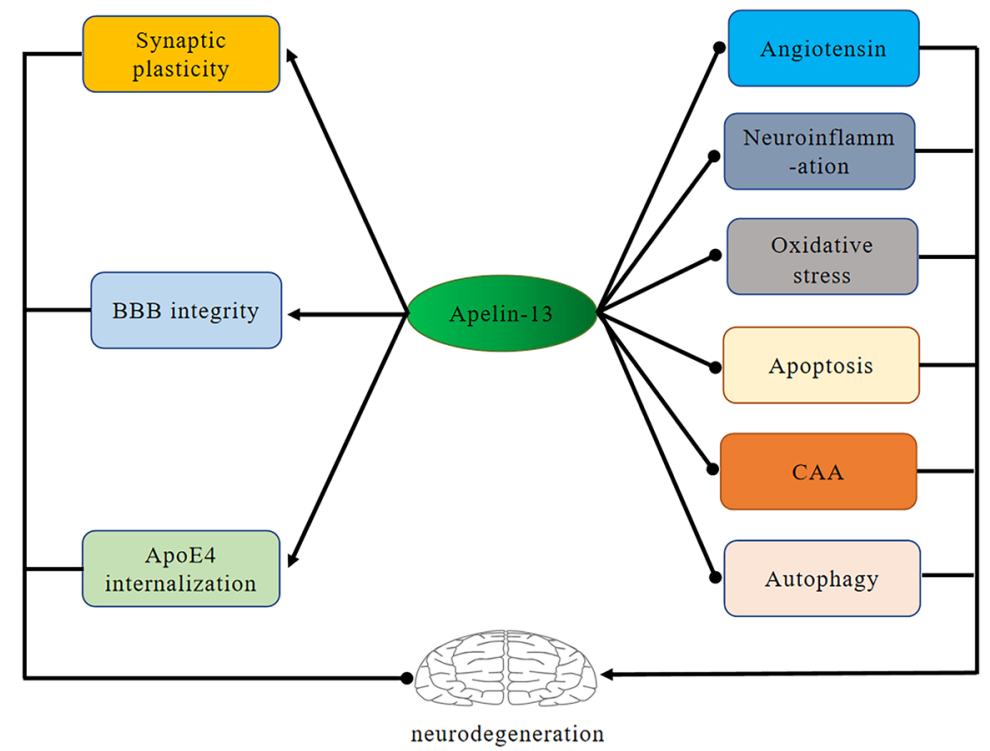

inhibition

$\underset{\text { promotion }}{\longrightarrow}$

\section{Apelin-13 and Apoptosis}

Apelin-13 inhibits rotenone and $A \beta$ induced injury and apoptosis in SH-SY5Y cells [38, 39]. It is also known that apelin-13 blocks apoptosis and excitotoxicity in mice with ischemic brain injury [40, 41]. Further, it has been found to increase cell survival under oxidative stress and decreased $\mathrm{Bax} / \mathrm{Bcl}-2$ ratio and caspase-3 protein's expression in antioxidant stress experiment of hair cell-like cells derived from bone marrow mesenchymal stem cells [42]. Moreover, apelin-13 alleviated the motor behavioral defects and inhibited dopaminergic neurodegeneration in 1-methyl-4-phenyl-1, 2, 3, 6-tetrahydropyridin (MPTP)-induced mice [43]. Inositol-requiring enzyme $\alpha$ (IRE1 $\alpha)$ is necessary and sufficient to trigger ERS. There are clinical trials illustrating that a feedback mechanism exists to attenuate IRE1 $\alpha$ ribonuclease activity in the presence of X-box binding protein 1 (XBP1) [44]. C/EBP homologous protein (CHOP) is important in ERS-induced apoptosis. Previous studies shows that apelin-13 also alleviated the activation of IRE1 $\alpha / \mathrm{XBP} 1 / \mathrm{CHOP}$ signaling pathway in MPTP-induced mice [45]. For instance, it has been shown to activates the Galpha (i)/Galpha (q)casein kinase 2 (CK2) signaling pathway and attenuates eukaryotic translation initiation factor 2 (eIF2)-activating transcription factor 4 (ATF4)-CHOP mediated apoptosis of neurons in ischemic stroke mice [46].

Glucagon-like peptide-1 receptor (GLP-1R) is a member of the $G$ protein coupled receptor superfamily which is widely expressed in neurons [47]. The inhibitory effect of GLP-1R on neuronal apoptosis has been reported in the case of subarachnoid hemorrhage (SAH). In SAH mice, GLP-1R expression dramatically increased and reached its highest peak in $24 \mathrm{~h} \mathrm{[48].} \mathrm{Further,} \mathrm{apelin-13} \mathrm{treatment} \mathrm{upregulated}$ the expression of GLP-1R, which inhibited cortical neuronal apoptosis. However, LY294002 [inhibitor of phosphoinositide 3-kinase (PI3K)/the serine/threonine kinases/Akt signaling pathway] andGLP-1R siRNA partly reversed the neuroprotective effects of apelin-13 [49]. Therefore, apelin-13 plays a critical role in neuroprotection and anti-AD properties by inhibiting the neuronal apoptosis induced by $\mathrm{A} \beta$ or others.

\section{Alzheimer's Disease, Apelin-13 and Autophagy Promotion}

\section{$A D$ and Autophagy}

To the best of our knowledge, autophagy involves productive and degradative process of $A \beta$ and tau protein. Normally, autophagy promotes the degradation and clearance of $A \beta$ and APP [50, 51]. Autophagy lysosome system not only degrades and clears $A \beta$, but also produces $A \beta$ under pathological or aging conditions [52]. It has been described that $\mathrm{A} \beta$ production is detected in immature autophagic vacuoles and aggregated immature autophagic vacuoles are found in the brain of AD mice [53, 54]. This suggests that aggregated immature autophagic vacuoles may be one source of increased $\mathrm{A} \beta$ in $\mathrm{AD}$. Therefore, inhibiting the formation of immature autophagic vacuoles may be an effective way of arresting the progression of AD. In addition, autophagy is responsible for the extracellular release of $A \beta$ [55]. It is postulated that many vacuoles with membranous structure will be manufactured in the course of autophagy. Immature vacuole requires to be modified by autophagy-related molecules to convert into a mature vacuole. The mature vacuoles fuses with lysosome and then initiates the degradation process [56]. However, accumulated immature vacuoles due to excessive production and interruption of transformation 
process chooses to fuse with cell membrane and then release the contents [57]. Autophagy is also involved in degradation of tau. Several studies have shown that the dysfunction of autophagy lysosome system leads to the formation of oligomers and insoluble polymers of tau, but the promotion of autophagy reverses this outcome [58]. Furthermore, deletion of crucial autophagy components such as ATG5 or ATG7 in the neurons of mice causes behavior deficits and neurodegeneration [59]. However, research has revealed that role of autophagy in $\mathrm{A} \beta$ plague and tangles degradation was limited. Autophagy performs its beneficial effects on AD only before the stable plagues and tangles are formed [60, 61]. In conclusion, autophagy plays a dual role in $\mathrm{A} \beta$ regulation and its harmful effects seem to be more predominant because of its weak ability to degrade $A \beta$.

\section{Apelin-13 and Autophagy}

There are several discrepancies regarding the relationship between autophagy and apelin-13. In H9C2 cells, apelin-13 stimulates autophagic vesicles, autophagy lysosome formation and increased expression of the autophagy markers Beclin-1 and LC3II/I [62]. In NP cells under oxidative stress, apelin-13 promotes autophagic flow, as evidenced by an increase in LC3II/I and a decrease in p62 [63]. In tumor cells A549 cells, apelin-13 has been found to induce autophagy by activating ERK1/2 signaling [64]. Apelin-13 (APJ) reduces the degeneration of dopaminergic neurons in the substantia nigra and striatum of rotenone-treated mice through activation of AMP-activated protein kinase (AMPK)/mammalian target of rapamycin (mTOR)/UNC-51-like kinase (ULK) 1 signaling pathway mediated autophagy [39]. It has also been shown that apelin-13 activates cardiomyocyte autophagy by acting on the PI3K/Akt pathway [65]. Further, the effect of apelin-13 on MPTP-induced neurotoxicity was inhibited [45]. Therefore, these studies suggest that apelin-13 contributes to autophagy. However, it has also been shown that apelin-13 has an inhibitory effect on autophagy. Expression levels of Beclin-1 and LC3II In METH-induced PC-12 cells, were significantly increased compared to controls. It was found that apelin-13 pretreatment significantly reduced the expression of these two proteins [66]. In I/R injury model, apelin-13 inhibited autophagy by activating the PI3K/Akt/ mTOR signaling pathway. The use of LY294002 attenuated the inhibitory effect of apelin-13 on autophagy [67].

Apelin-13 has both promotive and inhibitory effects on autophagy and studies have hence suggested that apelin-13 has a heterogeneous effect on the regulation of autophagy. Furthermore, this heterogeneity may be related to the cell line selected for the experiment, the animal model, the mode of administration, the type of agent, the dose of the agent and many other confounding factors. A different study has reported that $A \beta$ significantly increases the escape latency and reduce traveled distance, swimming speed and time spent in target quadrant in $\mathrm{AD}$ model of rat with $\mathrm{A} \beta 25$ 35 injected in the hippocampal CA1 region. The ratio of $\mathrm{mTOR} / \mathrm{mTOR}$ and LC3II/I ratio increased. However, the use of apelin-13 significantly reversed these results and reduced AD symptoms [26]. In conclusion, apelin-13 exerts an anti-AD effect by inhibiting autophagy. However, studies directly demonstrating the relationship between apelin-13 and autophagy in AD models are still lacking and the circumstances in which apelin-13 promotes autophagy in $\mathrm{AD}$ remain to be further elucidated.

\section{Alzheimer's Disease, Apelin-13 and Improvement on Synaptic Plasticity}

\section{Alzheimer's Disease and Synaptic Plasticity}

Accumulating findings suggests that synaptic loss and dysfunction are important causes of cognitive function impairment in patients with $\mathrm{AD}$ [68]. Long term potentiation (LTP), the most common type of synaptic plasticity, is the basis of learning and memory. Dopamine (DA) improves synaptic plasticity, LTP induction and structural learning by acting on dopaminergic receptor (DR) $1[69,70]$. $N$-methyl D-aspartate receptors (NMDARs) play an essential role in neuronal synaptic plasticity through maintenance of neuronal excitability, $\mathrm{Ca}^{2+}$ influx and memory formation [71]. NMDARs has high permeability to $\mathrm{Ca}^{2+}$ and mediates postsynaptic $\mathrm{Ca}^{2+}$ influx [72]. Under normal conditions, NMDARs perform its protective role in synaptic plasticity through LTP and long-term depression (LTD), separately induced by $\mathrm{Ca}^{2+} /$ calmodulin-dependent protein kinase II (CaMKII) and phosphatases [73]. Nonetheless, excessive accumulation of $\mathrm{Ca}^{2+}$ induces activation of nitric oxide synthase (NOS) and phospholipase A2 (PLA2). This incurs production of ROS and reactive nitrogen species (RNS) and eventually causing synaptic impairment and cell death [74]. This excitotoxic course triggered by over-secreted glutamate may be the potential mechanism of AD.

\section{Apelin-13 and Synaptic Plasticity}

Apelin-13 is a predominant neuropeptide with inhibiting effect on synaptic plasticity and has pivotal effects on cognition memory as well as neuronal protection [75]. Previous studies have shown that apelin-13 reduces 6-hydroxydopamine induced hippocampal early long-term potentiation (E-LTP) damage. It also induces the decrease of hippocampal synaptic proteins such as post synaptic density protein and DR1 [70]. Moreover, apelin-13 activates soluble guanylate cyclase and increases the level of cyclic guanosine monophosphate (cGMP) through endothelial nitric oxide (eNO) signal pathway and then improve synaptic plasticity 
[76]. Apelin-13 improves the synaptic plasticity by decreasing the excitotoxicity induced by overactivated NMDARs [77]. To protect neurons from NMDAR-dependent excitotoxicity, apelin-13 activates cell survival signals in neurons through IP3, PKC, MEK1/2, Raf/extracellular signal-regulated kinase 1/2 (ERK1/2) and AKT signaling pathways. On the other hand, it inhibits excitotoxicity by inhibiting the activity of NMDARs [78]. In conclusion, apelin-13 improves synaptic plasticity and protect neurons from excitotoxicity, which may improve the symptoms of $\mathrm{AD}$.

\section{Alzheimer's Disease, Apelin-13 as a Neuroinflammation Inhibitor}

\section{Microglia, Neuroinflammation and AD}

That the inflammatory markers are elevated in patients with $\mathrm{AD}$, suggesting that neuroinflammation may be an important driver of AD [79]. Microglial M1/M2 polarization, $\mathrm{A} \beta$ deposition and the cytokines network play a determinant role in inflammatory environment regulation associated with neurodegeneration $[80,81]$. Microglia play a predominant role in the neuroinflammation regulation by switching into a M1 or M2 phenotype promoting and curbing the inflammation respectively [82]. The stimulation of triggering receptor expressed in myeloid cells 2 (TREM2), a cell surface receptor on microglia, initiates signal transduction pathways that promote microglia chemotaxis, phagocytosis, survival and proliferation $[83,84]$. It is thought that the TREM2-APOE axis works in the microglia clearance of $A \beta$ aggregation and thus may be important for neuronal protection in $\mathrm{AD}$ [85]. TREM2 deficiency augment $A \beta$ and Tau accumulation due to a dysfunctional response of microglia [86, 87]. When toxic $A \beta$ and Tau accumulates in the stressed or damaged neurons, they induce microglia to enter the M1 proinflammatory state, where they devour synapses, secrete neurotoxic cytokines that damage neurons and contribute to AD [88]. A $\beta$ stimulates Toll-like receptors (TLRs) and the NRLP3 inflammasome leading to microglia production of tumor necrosis factor-alpha (TNF- $\alpha$ ), interleukin (IL)- $1 \beta$ and other inflammatory cytokines [89]. Apolipoprotein E (APOE) 4's toxic effects in the context of tau pathology are correlational with increased TNF- $\alpha$ production by microglia in vitro [90]. In $\mathrm{AD}$, amyloid protein, a neurotoxic substance, induces the activation of astrocytes and microglia after ischemia. This results in to injury and death of general neurons and glial cells [91]. Further, it has been shown that $\mathrm{A} \beta$ stimulates the microglia transforming into a pro-inflammatory phenotype [92]. Therefore, $A \beta$-induced neuro-inflammation may be a crucial mechanism for AD.

Recent genetic studies have provided overwhelming evidence of targeting microglia polarization that may be an effective avenue for neuroprotection and reducing neurological impairment in $\mathrm{AD}$ [93]. Curcumin reduces the imbalance of triggering receptor expressed on myeloid cells 2 and toll-like receptor 4 by transforming M1 microglia into M2. It also attenuates the activation of downstream nuclear factor-kappa B (NF- $\mathrm{kB})$, which significantly reduces the LPS-induced inflammatory response and plays a neuroprotective role [94]. Several findings suggest that microglia may always, directly or indirectly cause some "collateral damage" to neighboring neurons while performing their clearing function after being activated into M1 phenotype [95]. Upon activation of M1 microglia, the inflammatory cascade is triggered by the release of TNF- $\alpha$, IL- 6 and other proinflammatory molecules [96, 97]. Therefore, these changes lead to amyloid aggregation, tau formation, synaptic damage, neuronal loss and progression to AD [98]. A different study has indicated that as AD progresses, pro-inflammatory cytokines are produced in response to $A \beta$ deposition. The cytokines down-regulate genes in response to $A \beta$ clearance and decrease expression of the A beta-binding scavenger receptors (scavenger receptor A (SRA), CD36, the A betadegrading insulysin, neprilysin) therefore contributing to $A \beta$ accumulation $[99,100]$. Therefore, the imbalance between M1/M2-polarized microglia in healthy brain causes neuronal degeneration and hence AD-related pathological changes [101]. In conclusion, the pro- or anti-inflammatory process mediated by different cells (such as microglia and astrocytes) at different stages of $\mathrm{AD}$ may be harmful or beneficial to the organism.

\section{Apelin-13 and Neuroinflammation Within AD}

Apelin-13has beneficial properties on memory impairment and neuronal injury associated with AD [102]. Treatment with apelin-13 inhibits the increase of myeloperoxidase activity, decreased the expression of inflammatory cytokines such as TNF- $\alpha$ and intercellular adhesion molecule-1 (ICAM-1) and significantly reduced the neurological deficit and infarct volume in $\mathrm{I} / \mathrm{R}$ rats [103]. It has been reported that non-invasive intranasal administration of apelin-13 in poststroke mouse model, inhibits the inflammatory activity of ischemic brain [104]. Neuro-inflammation plays an important role in early brain injury after SAH. On the other hand, apelin-13 performs a neuroprotective role by inhibiting the activation of microglia and the neuroinflammation caused by endoplasmic reticulum stress [105]. Apparently, it was found that in the cases of cerebral infarcts, apelin-13 treatment improved the prognosis of nervous system, reduced brain edema and inhibited apoptosis and neuro-inflammation [106]. Furthermore, correlative studies have reported that apelin-13 inhibits the expression of pro-inflammatory M1 microglial marker CD86 and promote the expression of anti-inflammatory M2 microglial marker CD206 [107]. Apelin-13 inhibits the activation of microglia and astrocytes, 
decreases the expression of IL- $1 \beta$ and TNF- $\alpha$. It reduces the expression defect of brain-derived neurotrophic factor (BDNF)/ tyrosine receptor kinase B (TrkB) in the hippocampus and improves the cognitive deficits in streptozotocininduced $\mathrm{AD}$ phenotype rats. Elsewhere, it was found that apelin-13 mediated effect blocked by TrkB receptor antagonist K252A [108]. Overall, various research studies have shown that apelin-13 promotes the M2 microglia polarization but prevents the M1 microglia polarization, which is expected to become a new target of anti-AD therapy.

\section{Alzheimer's Disease, Apelin-13 and Oxidative Stress}

\section{Alzheimer's Disease and Oxidative Stress}

Previous studies have shown that at least two events, including decreased energy metabolism and increased oxidative stress in the brain caused by mitochondrial dysfunction, are characteristics of $\mathrm{AD}$ [109]. Oxidative stress is associated with protein oligomerization and mitochondrial dynamics leading to a vicious cycle of mitochondria and endoplasmic reticulum (ER) impairment as well as neurodegeneration [110]. The disorder of cell ion homeostasis and substance metabolism caused by $A \beta$ oxidative stress makes neurons prone to apoptosis. $\mathrm{A} \beta$ aggregation interacts with $\mathrm{Fe}^{2+}$ or $\mathrm{Cu}^{2+}$ to produce $\mathrm{H}_{2} \mathrm{O}_{2}[10]$. When $\mathrm{A} \beta$ aggregates on the cell membrane, membrane-related oxidative stress leads to lipid peroxidation and ER dysfunction [111]. Cupper ions in A $\beta$ are electrochemically active and promoting the production of ROS. These ROS leads to lipid peroxidation of neuronal cell membranes and malfunction of glucose transporters and ion channel ATPase, terminally contributing to cell injury or death [112]. Glycogen synthase kinase 3(GSK-3) is a serine/ threonine protein kinase, which participates in a variety of cellular activities, including apoptosis and the regulation of oxidative stress, and the pathophysiology of AD [113, 114]. Oxidative stress activates glycogen synthase kinase 3(GSK3 ) and c-Jun $N$-terminal kinase (JNK)/mitogen-activated protein kinases (MAPK) and hence increases NFTs and A $\beta$ by, respectively. This causes tau phosphorylation and $\beta$-site APP-cleaving enzyme $1 / \beta$-secretase (BACE1) expression $[5,115]$.

\section{Apelin-13 and Oxidative Stress Within AD}

Apelin-13 is a novel adipocytokine and is also known to be a powerful antioxidant. The distribution of apelin and APJ suggests that apelin-13 may be a key mediator in the development of stress-related behavior [116]. Transcription factor nuclear factor erythroid 2-related factor 2 (Nrf2) regulates the gene expression of anti-oxidation and anti-inflammation cytoprotective protein and plays an important role in oxidative stress [117]. It has been found that long-term exposure to methamphetamine (METH) triggers mental disorders. However, apelin-13 reduces METH-induced neurotoxicity by reducing oxidative stress, apoptosis and autophagy [66]. AMPK is a cellular energy receptor that plays a protective role in global cerebral ischemia [118]. Further, apelin-13 has been reported to induce the expression of antioxidant protein through AMPK/P-GSK-3/Nrf2 pathway, which also protects PC12 cells from I/R induced oxidative stress [106]. It plays an anti-depressant role and improves recognition and memory by activating PI3K and ERK1/2 signaling pathways in stress depressed and memory impaired rats [119]. Oxidative stress plays a crucial role in early brain injury after SAH. Apelin-13 reduces oxidative stress after SAH and early brain injury through AMPK/thioredoxin interacting protein/ NLRP3signal pathway [105]. Additionally, reports about the role of apelin-13 in SH-SY5Y cells treated with $A \beta$ have demonstrated that apelin- 13 evidently reduced the generation of intracellular ROS and $\mathrm{Ca}^{2+}$ at the dose of 2.5/5.0 ug/ $\mathrm{ml}$ [38]. Therefore, apelin-13-mediated antioxidant stress is a critical mechanism of anti-AD.

\section{Alzheimer's Disease, Apelin-13 and Blood Brain Barrier Protection}

Dysfunction blood brain barrier protection directly leads to decreased clearance of $A \beta$ and reduced cerebral plasma albumin and glucose uptake, leading to continued deterioration of AD [120]. Several studies have shown that apelin-13 plays a role in anti-ECs dysfunction by promoting vascular ECs proliferation and reducing vascular endothelial apoptosis [121]. Apelin-13/APJ system promotes vascular EC proliferation endothelium repair by activating eNOS, AMPK, ERK1/2/PI3K/P70S6K, PI3K/AKT, MAPK [22]. Hypoxiainduced ROS production causes Endoplasmic Reticulum (ER) stress, which leads to apoptosis of ECs, endothelial dysfunction and release of neurotoxic substances [122]. Apelin-13 reduces the apoptosis of ECs and help neurons in avoiding neurotoxic injury by up-regulating the level of AMPK and inhibiting ER stress [123]. Astrocyte is a key composition of BBB which may have an effect on the clearance of $A \beta$ [59]. A previous study has shown that apelin-13 reduces the apoptosis of astrocytes, promotes angiogenesis and thus protecting the BBB [124]. Another study showed that apelin-13 also significantly increased aquaporin-4 (AQP4) expression, decreased BBB permeability, increased vascular endothelial growth factor, upregulated eNO synthase, down-regulated inducible NOS, decreased neurological function score and reduced infarct volume [125]. Cerebrovascular ECs is part of BBB and hence the apelin/APJ system maintains the integrity of BBB. In addition, reduced ECs stress reduces the release of neurotoxic substances [104, 126]. Therefore, it has been described that apelin-13 may be a promising therapeutic target for AD by maintaining 
the structural and functional integrity of BBB and vascular endothelium.

\section{Alzheimer's Disease, Apelin-13 and Cerebral Amyloid Angiopathy Inhibition}

About $80 \%$ of patients with AD experience cerebrovascular amyloid angiopathy (CAA) [127]. Previous studies have shown that CAA is associated with cognitive decline in AD patients [128]. During the pathological process of CAA, uncleared $A \beta$ from the brain accumulates on arterial and arteriole walls, causing decreased capillary stretch, vascular degeneration, vascular stenosis as well as occlusion. Further development of CAA leads to BBB damage, microischemia and cerebral infarction $[129,130]$. The activities of $\beta$ - and $\gamma$-secreting enzymes are increased under hypoxia and ischemia, resulting to an increase in the production of APP [131]. At the same time, the damage of BBB blocked the clearance of neurotoxic substances such as APP and $\mathrm{A} \beta$, which further led to the formation of amyloid plaques, massive loss of neurons as well as brain atrophy, and hence exacerbate the performance of $\mathrm{AD}$ [132]. This suggests that the abnormal deposition of $A \beta$ in patients with $A D$ leads to local CAA lesions, which further aggravates the pathological progress of $\mathrm{AD}$. Cerebrovascular amyloid angiopathy (CAA) pathogenesis is depressed by eNO which plays a promoting role in vascular elasticity, expression of $A \beta$ transporter and $A \beta$ clearance [133]. Apelin-13 increases the expression of eNO, thus promoting A $\beta$ clearance [5]. Therefore, apelin-13 is considered as a therapeutic target for CAA and related disorders and may promote $A \beta$ clearance while reversing endothelial dysfunction.

\section{Alzheimer's Disease, Apelin-13 and Apolipoprotein E}

Apolipoprotein Es (APOEs) in the brain is mainly synthesized and secreted by astrocytes as well as microglia and participates in the lipid metabolism of neurons [134]. They are divided into APOE2, APOE3, APOE4 and other phenotypes. After lipidation with cholesterol transported across cell membrane by ATP-binding cassette transporter (ABC) A1 or ABCG1, APOEs acquire the ability to internalize into cells expressing corresponding receptors [135]. Many reports have shown that APOE is involved in the regulation of $\mathrm{AD}$ pathogenesis. Apolipoprotein $\mathrm{E}$ gene abnormalities are commonly seen in late-onset AD. The e 4 allele of APOE is related to the pathogenesis and severity of $\mathrm{AD}$ [136]. Apolipoprotein Es directly interacts on soluble $A \beta$ and $\mathrm{A} \beta$ fibrils [137]. Among them, APOE2 is considered to be beneficial for $\mathrm{AD}$ [138]. It promotes $\mathrm{A} \beta$ aggregation in interstitial fluid and prevents $A \beta$ clearance as compared with APOE2 and APOE3 [139]. ATP-binding cassette transporter A1 (ABCA1) and ABCG1 agonists enhances the lipidation of APOE4 by promoting the cholesterol efflux and promotes the internalization of APOE4 and thus reduces the level of $A \beta$ in interstitial fluid [140]. So far, a few studies have reported that there may be a signal pathway of apelin-13 acting on APOE. Apelin-13 increases the level of ABCA1 protein by activating protein kinase $\mathrm{C} \alpha(\mathrm{PKC} \alpha)$ and inhibiting calmodulin-induced ABCA1 degradation in THP-1 macrophage-derived foam cells [141]. This suggests that apelin-13 can promotes the internalization of APOE4, thus promotes $A \beta$ clearance and inhibits the formation of pathological $A \beta$ plaques and finally depresses the progression of AD. Further experimental studies should be carried out to clarify whether apelin-13 acts on APOE2 and APOE3. Further studies should also be carried out to ascertain whether apelin-13 can act on other regulatory signals like ABCG1 to promote the lipidation of APOE4.

\section{Alzheimer's Disease, Apelin-13 and Angiotensin}

Studies show that upregulation of angiotensin II (Ang II) aggravates $\mathrm{AD}$ progression through its effects on cerebral blood flow [142]. Ang II promotes expression of GSK3 $\beta$, APP and increases BACE1 activity, thus upregulating p-tau and $A \beta[143,144]$. Evidence from previous studies has shown that apelin-13 inhibits Ang II signaling pathway, promotes the conversion of Ang II to angiotensin 1-7 with memory and cognitive enhancing function, and reduces production of APP [5]. It has been reported that apelin-13/ APJ combines with Ang II type-1 receptor (AT1) to form an APJ-AT1 heterodimer, which prevents the Ang II from binding to AT1 [145]. Apelin-13 also inhibits BACE1 in PC-12 cell line and reduces the formation of $A \beta$ [5]. Inhibition of IRE1 $\alpha /$ XBP1/CHOP signaling pathway has been shown to mediate the neuroprotective effects of Telmisartan, one unique Ang II type 1 receptor blocker. This indicates that Ang II induces neuronal apoptosis in an IRE1 $\alpha / \mathrm{XBP} 1 /$ CHOP-dependent manner [146]. Uric acid can induce oxidative stress by activating renin-angiotensin system (RAS) in 3T3-L1 adipocytes. A study showed that intraperitoneal injection of apelin-13 for 12 weeks in rats with hyperuricemia decreased oxidative stress, down-regulated RAS levels, and up-regulated APJ expression in adipose tissue [147]. Angiotensin also mediates the inhibitory effects of apelin-13 on $\mathrm{A} \beta$ production.

\section{Summary and Future Prospects}

Recent epidemiological studies have shown that AD occurs in patients with dementia. Although the currently used drugs slows down the progression of $\mathrm{AD}$, they are not effective in preventing neuronal damage. Therefore, there is a need to develop new drugs for AD. Studies have shown that abnormal 


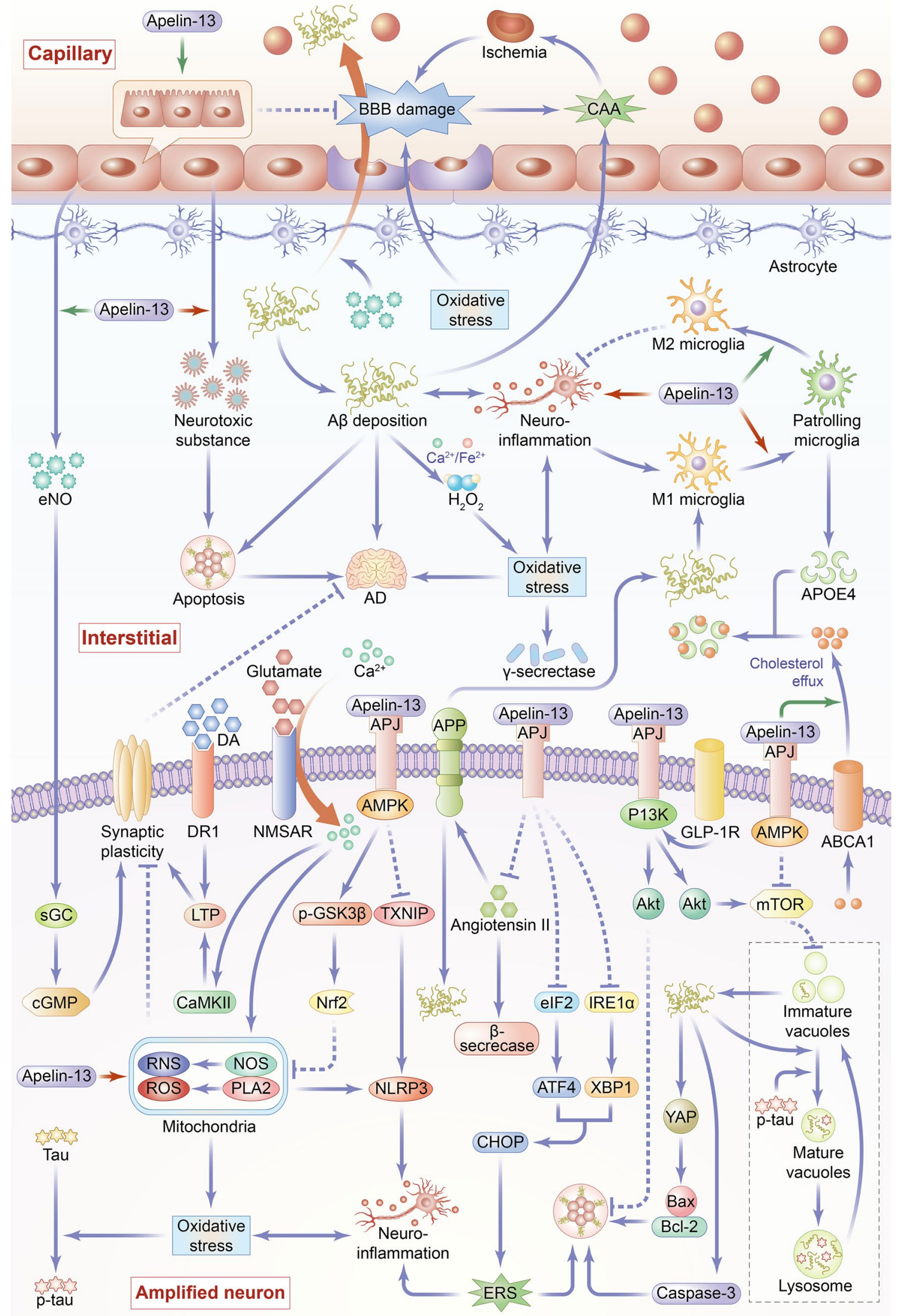


4Fig. 2 Overview of the main mechanisms and signaling pathways of Apelin-13 in regulating Alzheimer's disease. The pathogenesis of AD is complex and diverse, and its occurrence and development are closely related to many factors, such as increased apoptosis, enhanced autophagy, synaptic plasticity disorder, elevated level of neuroinflammation, increased oxidative stress, destruction of blood brain barrier (BBB), formation of cerebrovascular amyloid angiopathy (CAA), and unesterified apolipoprotein $\mathrm{E}$ (APOE4). Apelin-13 plays an anti-AD role through the following mechanisms. 1. Apoptosis (1) Apelin-13 inhibits the production and aggregation of $\beta$-amyloid protein $(A \beta)$, whereas $A \beta$ induces apoptosis by regulating the Yes-related proteins-related protein (YAP) to increase the $\mathrm{Bax} / \mathrm{Bcl}-2$ ratio and promote caspase-3 protein expression. (2) Apelin-13 inhibits the cell apoptosis by promoting Glucagon-like peptide-1 receptor (GLP-1R) expression. (3) Apelin-13 enhances phosphoinositide 3-kinase (PI3K)/Akt signaling pathway to inhibit neuronal apoptosis. (4) Apelin-13 reduces endoplasmic reticulum stress (ERS) by inhibiting inositol-requiring enzyme $\alpha$ (IRE-1 $\alpha) / \mathrm{X}$-box binding protein 1 (XBP1)/C/ EBP homologous protein (CHOP) and eukaryotic translation initiation factor 2 (eIF2)/activating transcription factor 4 (ATF4)/CHOP signaling pathways, thereby inhibiting cell apoptosis. 2. Autophagy Apelin-13 paly a both promoting and inhibiting roles in autophagy through activation of (AMP-activated protein kinase) AMPK/(mammalian target of rapamycin) mTOR/(UNC-51-like kinase 1) ULK1 signaling pathway and of PI3K/ Akt/mTOR signaling pathway. Apelin-13 exerts its anti-AD effects mainly through the inhibition of cellular autophagy, which inhibits the formation of immature autophagy follicles and produce of $\mathrm{A} \beta$. 3. Synaptic plasticity (1) Apelin-13 activates soluble guanylate cyclase (sGC) and increases the level of cyclic guanosine monophosphate (cGMP) through endothelial nitric oxide (eNO) signal pathway then improves synaptic plasticity. (2) Normally, $\mathrm{N}$-methyl D-aspartate receptors (NMDAR) induce long term potentiation to improve synaptic plasticity. Apelin-13 inhibits oxidative stress induced by overactivated NMDAR, thereby reducing synaptic damage and improving synaptic plasticity. (3) Apelin-13 increases dopaminergic receptor 1 (DR1) levels and promotes DA-induced LTP, thereby promoting synaptic plasticity. 4. Neuroinflammation (1) Apelin-13 exerts an anti-inflammatory role by promoting microglia polarization to M2 phenotype and inhibiting microglia polarization to M1 phenotype. (2) Apelin-13 reduces reactive oxygen species (ROS)-induced oxidative damage in mitochondria and inflammatory response induced by nucleotide-binding and oligomerization domain-like receptor (NLR) family pyrin domain-containing 3 inflammasome activation. (3) Apelin-13 reduces ERS, thus inhibiting neuroinflammation. 5. Oxidative stress (1) Apelin-13 inhibits NMDAR activity, sequentially inhibiting the production of ROS and reactive nitrogen species caused by excessive calcium ion influx. (2) Apelin-13 inhibits $A \beta$ aggregation, which interacts with $\mathrm{Fe} 2+$ or $\mathrm{Cu} 2+$ to produce $\mathrm{H} 2 \mathrm{O} 2$ which induces oxidative stress. (3) Apelin-13 induces the expression of antioxidant protein through AMPK/PGSK-3 $\beta$ /nuclear factor erythroid 2-related factor 2 (Nrf2) pathway or AMPK/thioredoxin interacting protein (TXNIP)/NLRP3 signal pathway, which relive oxidative stress-related cell damage. 6. BBB (1) Apelin-13 promotes vascular endothelial cell proliferation. (2) Apelin-13 inhibits oxidative stress-mediated BBB damage. 7. CAA (1) Apelin-13 promotes endothelial nitric oxide (eNO) secretion by vascular endothelium, and thus enhances the ability of vascular transmembrane transport to $A \beta$, thereby promoting the clearance of $\mathrm{A} \beta$ and inhibiting CAA. (2) Apelin-13 has an inhibitory effect on CAA by inhibiting the increase of $\beta$ and $\gamma$ secretase activity mediated by oxidative stress. (3) Apelin-13 inhibits the angiotensin II signaling pathway, thereby decreasing the activity of $\beta$-site APPcleaving enzyme $1 / \beta$-secretase and inhibiting the generation of amyloid precursor protein (APP), playing an inhibitory effect on CAA. (4) Apelin-13 promotes the internalization of APOE4 that reduces the level of A $\beta$ in intertissue-fluid, thus inhibiting CAA. 8. APOE4 Apelin-13 promotes the expression of ATP-binding cassette transporter A1 (ABCA1) protein, which facilitates cholesterol outflow to promote the lipidation of APOE, and thus enhances APOE4 internalization levels of CAA and APOE, deposition of A $\beta$ and tau, neuroinflammatory responses by microglia and astrocytes, ischemia, hypoxia, and NFTs contributes to the development of AD.

The data reviewed here demonstrate that apelin-13 can be a therapeutic target for AD management. Apelin-13 participates in the regulation of autophagy, apoptosis, synaptic plasticity of neurons and polarization of microglia thereby slowing AD progression. Apelin-13 protects neurons by decreasing oxidative stress, neuroinflammation, and blocking Ang II-related signaling pathways. In addition, it inhibits CAA and promotes APOE4 internalization, both of which reduce $A \beta$ deposition in the stroma, neurodegeneration and AD progression (Fig. 2).

Although apelin-13 seems to confer therapeutic benefits in AD, it may cause unexpected side effects given its widespread expression on blood vessels and diverse cellular activities. Moreover, the mechanisms regulating apelin-13 expression in brain tissue are not fully understood. Similarly, the mechanism by which apelin-13 influences polarization of microglia are unclear. Whether, apelin-13 play similar roles in Parkinson's disease, Huntington's disease and AD remain to be clarified. Further research is advocated to resolve these questions and provide new ideas for the prevention and treatment of AD and similar diseases.

Acknowledgements Not applicable.

Author Contributions TW and MF wrote the manuscript. WJ and PL prepared figures. YJ and SZ critically revised the manuscript for important intellectual content. All authors read and approved the final manuscript and agree to be accountable for all aspects of this work. Data authentication is not applicable.

Funding This study was supported by grants from the National Natural Science Foundation of China (Grant No. 82060250), the Guangxi Science and Technology Plan Project (Grant No. AD20238035), the Open Project of Guangxi Key Laboratory of Brain and Cognitive Neuroscience (Grant No. GKLBCN-20190105-02) and the Guangxi Zhuang Autonomous Region Students' Innovation and Entrepreneurship Training Program of China (Grant No. 202010601050).

Data Availability Not applicable.

\section{Declarations}

Conflict of interest The authors declare that they have no conflict of interest.

Ethics Approval and Consent to Participate Not applicable.

Patient Consent for Publication Not applicable.

\section{References}

1. Wiley J (2021) 2021 Alzheimer's disease facts and figures. Alzheimers Dement 17:327-406 
2. Avila J, Perry G (2021) A multilevel view of the development of Alzheimer's disease. Neuroscience 457:283-293

3. Livingston G, Huntley J, Sommerlad A et al (2020) Dementia prevention, intervention, and care: 2020 report of the Lancet Commission. Lancet 396:413-446

4. Luo H, Han L, Xu J (2020) Apelin/APJ system: a novel promising target for neurodegenerative diseases. J Cell Physiol 235(2):638-657

5. Masoumi J, Abbasloui M, Parvan R et al (2018) Apelin, a promising target for Alzheimer disease prevention and treatment. Neuropeptides 70:76-86

6. Yan J, Wang A, Cao J et al (2020) Apelin/APJ system: an emerging therapeutic target for respiratory diseases. Cell Mol Life Sci 77(15):2919-2930

7. Derby CA (2020) Trends in the public health significance, definitions of disease, and implications for prevention of Alzheimer's disease. Curr Epidemiol Rep 7:68-76

8. DeTure MA, Dickson DW (2019) The neuropathological diagnosis of Alzheimer's disease. Mol Neurodegener 14:32

9. Liddelow SA, Guttenplan KA, Clarke LE et al (2017) Neurotoxic reactive astrocytes are induced by activated microglia. Nature 541:481-487

10. Abeysinghe A, Deshapriya R, Udawatte C (2020) Alzheimer's disease; a review of the pathophysiological basis and therapeutic interventions. Life Sci 256:117996

11. Gosztyla ML, Brothers HM, Robinson SR (2018) Alzheimer's amyloid-beta is an antimicrobial peptide: a review of the evidence. J Alzheimers Dis 62:1495-1506

12. Kent SA, Spires-Jones TL, Durrant CS (2020) The physiological roles of tau and Abeta: implications for Alzheimer's disease pathology and therapeutics. Acta Neuropathol 140:417-447

13. Vaz M, Silvestre S (2020) Alzheimer's disease: recent treatment strategies. Eur J Pharmacol 887:173554

14. Zhang XX, Tian Y, Wang ZT et al (2021) The epidemiology of Alzheimer's disease modifiable risk factors and prevention. J Prev Alzheimers Dis 8:313-321

15. Soria Lopez JA, Gonzalez HM, Leger GC (2019) Alzheimer's disease. Handb Clin Neurol 167:231-255

16. Breijyeh Z, Karaman R (2020) Comprehensive review on Alzheimer's disease: causes and treatment. Molecules 25(24):5789

17. Sharma P, Sharma A, Fayaz F et al (2020) Biological signatures of Alzheimer's disease. Curr Top Med Chem 20(9):770-781

18. Wu L, Chen L, Li L (2017) Apelin/APJ system: a novel promising therapy target for pathological angiogenesis. Clin Chim Acta 466:78-84

19. Antushevich H, Wójcik M (2018) Review: apelin in disease. Clin Chim Acta 483:241-248

20. Liu W, Yan J, Pan W et al (2020) Apelin/Elabela-APJ: a novel therapeutic target in the cardiovascular system. Ann Transl Med 8:243

21. Mughal A, O'Rourke ST (2018) Vascular effects of apelin: mechanisms and therapeutic potential. Pharmacol Ther 190:139-147

22. Cheng J, Luo X, Huang Z et al (2019) Apelin/APJ system: a potential therapeutic target for endothelial dysfunction-related diseases. J Cell Physiol 234:12149-12160

23. Li L, Zeng H, Chen JX (2012) Apelin-13 increases myocardial progenitor cells and improves repair postmyocardial infarction. Am J Physiol Heart Circ Physiol 303:H605-618

24. Zhou Q, Chen L, Tang M et al (2018) Apelin/APJ system: a novel promising target for anti-aging intervention. Clin Chim Acta 487:233-240

25. Vinel C, Lukjanenko L, Batut A et al (2018) The exerkine apelin reverses age-associated sarcopenia. Nat Med 24:1360-1371

26. Aminyavari S, Zahmatkesh M, Farahmandfar M et al (2019) Protective role of Apelin-13 on amyloid $\beta 25-35$-induced memory deficit; Involvement of autophagy and apoptosis process. Prog Neuropsychopharmacol Biol Psychiatry 89:322-334

27. Ge Y, Li Y, Chen Q et al (2018) Adipokine apelin ameliorates chronic colitis in Il-10-/- mice by promoting intestinal lymphatic functions. Biochem Pharmacol 148:202-212

28. Castan-Laurell I, Dray C, Attane C et al (2011) Apelin, diabetes, and obesity. Endocrine 40:1-9

29. Yang Y, Lv SY, Ye W et al (2016) Apelin/APJ system and cancer. Clin Chim Acta 457:112-116

30. Dray C, Knauf C, Daviaud D et al (2008) Apelin stimulates glucose utilization in normal and obese insulin-resistant mice. Cell Metab 8:437-445

31. Gourdy P, Cazals L, Thalamas C et al (2018) Apelin administration improves insulin sensitivity in overweight men during hyperinsulinaemic-euglycaemic clamp. Diabetes Obes Metab 20:157-164

32. Kalantaripour TP, Esmaeili-Mahani S, Sheibani V et al (2017) Apelin-13 protects rat primary cortical glia-neuron co-culture against pentylenetetrazole-induced toxicity. Biomed Pharmacother 87:661-668

33. Radi E, Formichi P, Battisti C et al (2014) Apoptosis and oxidative stress in neurodegenerative diseases. J Alzheimers Dis 42(Suppl 3):S125-S152

34. Obulesu M, Lakshmi MJ (2014) Apoptosis in Alzheimer's disease: an understanding of the physiology, pathology and therapeutic avenues. Neurochem Res 39:2301-2312

35. Zhang H, Wu S, Xing D (2011) YAP accelerates Abeta (25-35)-induced apoptosis through upregulation of Bax expression by interaction with p73. Apoptosis 16:808-821

36. Armstrong R (2019) Risk factors for Alzheimer's disease. Folia Neuropathol 57(2):87-105

37. Wu Y, Wang X, Zhou X et al (2017) Temporal expression of apelin/apelin receptor in ischemic stroke and its therapeutic potential. Front Mol Neurosci 10:1

38. Samandari-Bahraseman MR, Elyasi L (2021) Apelin-13 protects human neuroblastoma SH-SY5Y cells against amyloidbeta induced neurotoxicity: involvement of antioxidant and antiapoptotic properties. J Basic Clin Physiol Pharmacol. https://doi.org/10.1515/jbcpp-2020-0294

39. Chen P, Wang Y, Chen L et al (2020) Apelin-13 Protects dopaminergic neurons against rotenone-induced neurotoxicity through the AMPK/mTOR/ULK-1 mediated autophagy activation. Int J Mol Sci 21(21):8376

40. Zeng XJ, Yu SP, Zhang L et al (2010) Neuroprotective effect of the endogenous neural peptide apelin in cultured mouse cortical neurons. Exp Cell Res 316:1773-1783

41. Yang Y, Zhang XJ, Li LT et al (2016) Apelin-13 protects against apoptosis by activating AMP-activated protein kinase pathway in ischemia stroke. Peptides 75:96-100

42. Niknazar S, Abbaszadeh HA, Peyvandi H et al (2019) Protective effect of [Pyr1]-apelin-13 on oxidative stress-induced apoptosis in hair cell-like cells derived from bone marrow mesenchymal stem cells. Eur J Pharmacol 853:25-32

43. Jiang Y, Liu H, Ji B et al (2018) Apelin 13 attenuates ER stress associated apoptosis induced by MPP+ in SHSY5Y cells. Int J Mol Med 42:1732-1740

44. Chalmers F, van Lith M, Sweeney B et al (2017) Inhibition of IRE1alpha-mediated XBP1 mRNA cleavage by XBP1 reveals a novel regulatory process during the unfolded protein response. Wellcome Open Res 2:36

45. Zhu J, Dou S, Jiang Y et al (2019) Apelin-13 protects dopaminergic neurons in MPTP-induced Parkinson's disease model mice through inhibiting endoplasmic reticulum stress and promoting autophagy. Brain Res 1715:203-212 
46. Wu F, Qiu J, Fan Y et al (2018) Apelin-13 attenuates ER stressmediated neuronal apoptosis by activating Galphai/Galphaq-CK2 signaling in ischemic stroke. Exp Neurol 302:136-144

47. Alvarez E, Roncero I, Chowen JA et al (1996) Expression of the glucagon-like peptide-1 receptor gene in rat brain. J Neurochem 66:920-927

48. Xie Z, Enkhjargal B, Wu L et al (2018) Exendin-4 attenuates neuronal death via GLP-1R/PI3K/Akt pathway in early brain injury after subarachnoid hemorrhage in rats. Neuropharmacology 128:142-151

49. Liu Y, Zhang T, Wang Y et al (2019) Apelin-13 attenuates early brain injury following subarachnoid hemorrhage via suppressing neuronal apoptosis through the GLP-1R/PI3K/Akt signaling. Biochem Biophys Res Commun 513:105-111

50. Zhou F, van Laar T, Huang $\mathrm{H}$ et al (2011) APP and APLP1 are degraded through autophagy in response to proteasome inhibition in neuronal cells. Protein Cell 2:377-383

51. Son SM, Jung ES, Shin HJ et al (2012) Abeta-induced formation of autophagosomes is mediated by RAGE-CaMKKbeta-AMPK signaling. Neurobiol Aging 33(1006):e1011-e1023. https://doi. org/10.1016/j.neurobiolaging.2011.09.039

52. Yu WH, Cuervo AM, Kumar A et al (2005) Macroautophagya novel Beta-amyloid peptide-generating pathway activated in Alzheimer's disease. J Cell Biol 171:87-98

53. Nixon RA, Wegiel J, Kumar A et al (2005) Extensive involvement of autophagy in Alzheimer disease: an immuno-electron microscopy study. J Neuropathol Exp Neurol 64:113-122

54. Mizushima N (2005) A(beta) generation in autophagic vacuoles. J Cell Biol 171:15-17

55. Nilsson P, Saido TC (2014) Dual roles for autophagy: degradation and secretion of Alzheimer's disease Abeta peptide. BioEssays 36:570-578

56. Li Q, Liu Y, Sun M (2017) Autophagy and Alzheimer's disease. Cell Mol Neurobiol 37:377-388. https://doi.org/10.1007/ s10571-016-0386-8

57. Cho SJ, Yun SM, Jo C et al (2015) SUMO1 promotes A $\beta$ production via the modulation of autophagy. Autophagy 11(1):100-112

58. Sanchez-Varo R, Trujillo-Estrada L, Sanchez-Mejias E et al (2012) Abnormal accumulation of autophagic vesicles correlates with axonal and synaptic pathology in young Alzheimer's mice hippocampus. Acta Neuropathol 123(1):53-70

59. De Strooper B, Karran E (2016) The cellular phase of Alzheimer's disease. Cell 164:603-615. https://doi.org/10.1016/j.cell. 2015.12.056

60. Spilman P, Podlutskaya N, Hart MJ et al (2010) Inhibition of mTOR by rapamycin abolishes cognitive deficits and reduces amyloid-beta levels in a mouse model of Alzheimer's disease. PLoS ONE 5:e9979

61. Majumder S, Richardson A, Strong R et al (2011) Inducing autophagy by rapamycin before, but not after, the formation of plaques and tangles ameliorates cognitive deficits. PLoS ONE 6:e25416

62. Wu D, Xie F, Xiao L et al (2017) Caveolin-1-autophagy pathway mediated cardiomyocyte hypertrophy induced by apelin- 13 . DNA Cell Biol 36(8):611-618

63. Jiang W, Zhao P, Zhang X (2020) Apelin promotes ECM synthesis by enhancing autophagy flux via TFEB in human degenerative NP cells under oxidative stress. Biomed Res Int 2020:4897170. https://doi.org/10.1155/2020/4897170

64. Yang L, Su T, Lv D et al (2014) ERK1/2 mediates lung adenocarcinoma cell proliferation and autophagy induced by apelin-13. Acta Biochim Biophys Sin (Shanghai) 46(2):100-111. https:// doi.org/10.1093/abbs/gmt140

65. Xie F, Liu W, Feng F et al (2015) K1/2-p70S6K and PI3Kinduced autophagy. Acta Biochim Biophys Sin (Shanghai) 47(12):969-980. https://doi.org/10.1093/abbs/gmv111
66. Foroughi K, Khaksari M, Rahmati M et al (2019) Apelin-13 protects $\mathrm{PC} 12$ cells against methamphetamine-induced oxidative stress, autophagy and apoptosis. Neurochem Res 44:2103-2112

67. Shao ZQ, Dou SS, Zhu JG et al (2021) Apelin-13 inhibits apoptosis and excessive autophagy in cerebral ischemia/reperfusion injury. Neural Regen Res 16(6):1044-1051. https://doi.org/10. 4103/1673-5374.300725

68. Chen Y, Fu AKY, Ip NY (2019) Synaptic dysfunction in Alzheimer's disease: mechanisms and therapeutic strategies. Pharmacol Ther 195:186-198

69. Centonze D, Gubellini P, Picconi B et al (2001) An abnormal striatal synaptic plasticity may account for the selective neuronal vulnerability in Huntington's disease. Neurol Sci 22:61-62

70. Esmaeili-Mahani S, Haghparast E, Nezhadi A et al (2021) Apelin-13 prevents hippocampal synaptic plasticity impairment in Parkinsonism rats. J Chem Neuroanat 111:101884

71. Kamat PK, Kalani A, Rai S et al (2016) Mechanism of oxidative stress and synapse dysfunction in the pathogenesis of Alzheimer's disease: understanding the therapeutics strategies. Mol Neurobiol 53:648-661

72. Binvignat $\mathrm{O}$, Olloquequi $\mathrm{J}$ (2020) Excitotoxicity as a target against neurodegenerative processes. Curr Pharm Des 26(12):1251-1262

73. Wang R, Reddy PH (2017) Role of glutamate and NMDA receptors in Alzheimer's disease. J Alzheimers Dis 57:1041-1048

74. Sekerdag E, Solaroglu I, Gursoy-Ozdemir Y (2018) Cell death mechanisms in stroke and novel molecular and cellular treatment options. Curr Neuropharmacol 16(9):1396-1415

75. Lv SY, Chen WD, Wang YD (2020) The Apelin/APJ system in psychosis and neuropathy. Front Pharmacol 11:320. https://doi. org/10.3389/fphar.2020.00320

76. Bon CL, Garthwaite J (2003) On the role of nitric oxide in hippocampal long-term potentiation. J Neurosci 23:1941-1948

77. Cheng B, Chen J, Bai B et al (2012) Neuroprotection of apelin and its signaling pathway. Peptides 37(1):171-173

78. Cook DR, Gleichman AJ, Cross SA et al (2011) NMDA receptor modulation by the neuropeptide apelin: implications for excitotoxic injury. J Neurochem 118:1113-1123

79. Leng F, Edison P (2021) Neuroinflammation and microglial activation in Alzheimer disease: where do we go from here? Nat Rev Neurol 17:157-172

80. Regen F, Hellmann-Regen J, Costantini E et al (2017) Neuroinflammation and Alzheimer's disease: implications for microglial activation. Curr Alzheimer Res 14:1140-1148

81. Shen Z, Li X, Bao X et al (2017) Microglia-targeted stem cell therapies for Alzheimer disease: a preclinical data review. J Neurosci Res 95:2420-2429

82. Wang J, Xing H, Wan L et al (2018) Treatment targets for M2 microglia polarization in ischemic stroke. Biomed Pharmacother 105:518-525

83. Wang Y, Ulland TK, Ulrich JD et al (2016) TREM2-mediated early microglial response limits diffusion and toxicity of amyloid plaques. J Exp Med 213(5):667-675

84. Poliani PL, Wang Y, Fontana E et al (2015) TREM2 sustains microglial expansion during aging and response to demyelination. J Clin Invest 125(5):2161-2170

85. Atagi Y, Liu CC, Painter MM et al (2015) Apolipoprotein E is a ligand for triggering receptor expressed on myeloid cells 2 (TREM2). J Biol Chem 290(43):26043-26050

86. Wang Y, Cella M, Mallinson K et al (2015) TREM2 lipid sensing sustains the microglial response in an Alzheimer's disease model. Cell 160(6):1061-1071

87. Heneka MT, Golenbock DT, Latz E (2015) Innate immunity in Alzheimer's disease. Nat Immunol 16(3):229-236

88. Hansen DV, Hanson JE, Sheng M (2018) Microglia in Alzheimer's disease. J Cell Biol 217(2):459-472 
89. Heneka MT, Kummer MP, Stutz A et al (2013) NLRP3 is activated in Alzheimer's disease and contributes to pathology in APP/PS1 mice. Nature 493(7434):674-678

90. Shi Y, Yamada K, Liddelow SA et al (2017) ApoE4 markedly exacerbates tau-mediated neurodegeneration in a mouse model of tauopathy. Nature 549(7673):523-527

91. Minter MR, Taylor JM, Crack PJ (2016) The contribution of neuroinflammation to amyloid toxicity in Alzheimer's disease. J Neurochem 136:457-474

92. Cai Z, Hussain MD, Yan LJ (2014) Microglia, neuroinflammation, and beta-amyloid protein in Alzheimer's disease. Int J Neurosci 124:307-321

93. Condello C, Yuan P, Grutzendler J (2018) Microglia-mediated neuroprotection, TREM2, and Alzheimer's disease: evidence from optical imaging. Biol Psychiatry 83(4):377-387

94. Zhang J, Zheng Y, Luo Y et al (2019) Curcumin inhibits LPSinduced neuroinflammation by promoting microglial M2 polarization via TREM2/TLR4/NF-kappaB pathways in BV2 cells. Mol Immunol 116:29-37

95. Yang X, Xu S, Qian Y et al (2017) Resveratrol regulates microglia M1/M2 polarization via PGC-1 $\alpha$ in conditions of neuroinflammatory injury. Brain Behav Immun 64:162-172

96. Belkhelfa M, Rafa $H$, Medjeber $O$ et al (2014) IFN- $\gamma$ and $\mathrm{TNF}-\alpha$ are involved during Alzheimer disease progression and correlate with nitric oxide production: a study in Algerian patients. J Interferon Cytokine Res 34(11):839-847

97. Brosseron F, Krauthausen M, Kummer M et al (2014) Body fluid cytokine levels in mild cognitive impairment and Alzheimer's disease: a comparative overview. Mol Neurobiol 50(2):534-544

98. Kaur D, Sharma V, Deshmukh R (2019) Activation of microglia and astrocytes: a roadway to neuroinflammation and Alzheimer's disease. Inflammopharmacology 27(4):663-677

99. Hickman SE, Allison EK, El Khoury J (2008) Microglial dysfunction and defective beta-amyloid clearance pathways in aging Alzheimer's disease mice. J Neurosci 28(33):8354-8360

100. Heneka MT, Carson MJ, El Khoury J et al (2015) Neuroinflammation in Alzheimer's disease. Lancet Neurol 14(4):388-405

101. Hellmann-Regen J, Kronenberg G, Uhlemann R et al (2013) Accelerated degradation of retinoic acid by activated microglia. J Neuroimmunol 256:1-6

102. Fan J, Guang H, Zhang H et al (2018) SIRT1 mediates apelin-13 in ameliorating chronic normobaric hypoxia-induced anxiety-like behavior by suppressing NF- $\mathrm{KB}$ pathway in mice hippocampus. Neuroscience 381:22-34

103. Xin Q, Cheng B, Pan Y et al (2015) Neuroprotective effects of apelin-13 on experimental ischemic stroke through suppression of inflammation. Peptides 63:55-62

104. Chen D, Lee J, Gu X et al (2015) Intranasal delivery of apelin-13 is neuroprotective and promotes angiogenesis after ischemic stroke in mice. ASN Neuro 7(5):1759091415605114

105. Xu W, Li T, Gao L et al (2019) Apelin-13/APJ system attenuates early brain injury via suppression of endoplasmic reticulum stress-associated TXNIP/NLRP3 inflammasome activation and oxidative stress in a AMPK-dependent manner after subarachnoid hemorrhage in rats. J Neuroinflamm 16:247

106. Duan J, Cui J, Yang Z et al (2019) Neuroprotective effect of Apelin 13 on ischemic stroke by activating AMPK/GSK-3beta/ Nrf2 signaling. J Neuroinflamm 16:24

107. Zhou S, Guo X, Chen S et al (2019) Apelin-13 regulates LPSinduced N9 microglia polarization involving STAT3 signaling pathway. Neuropeptides 76:101938

108. Luo H, Xiang Y, Qu X et al (2019) Apelin-13 suppresses neuroinflammation against cognitive deficit in a streptozotocin-induced rat model of Alzheimer's disease through activation of BDNFTrkB signaling pathway. Front Pharmacol 10:395
109. Grill JD, Karlawish J (2017) Study partners should be required in preclinical Alzheimer's disease trials. Alzheimers Res Ther 9:93

110. Melo TQ, Copray S, Ferrari MFR (2018) Alpha-synuclein toxicity on protein quality control, mitochondria and endoplasmic reticulum. Neurochem Res 43:2212-2223

111. Xie H, Hou S, Jiang J et al (2013) Rapid cell death is preceded by amyloid plaque-mediated oxidative stress. Proc Natl Acad Sci USA 110(19):7904-7909

112. Mattson MP (2004) Pathways towards and away from Alzheimer's disease. Nature 430:631-639

113. Kirouac L, Rajic AJ, Cribbs DH et al (2017) Activation of RasERK signaling and GSK-3 by amyloid precursor protein and amyloid beta facilitates neurodegeneration in Alzheimer's disease. ENeuro. https://doi.org/10.1523/ENEURO.0149-16.2017

114. Lauretti E, Dincer O, Pratico D (2020) Glycogen synthase kinase-3 signaling in Alzheimer's disease. Biochim Biophys Acta Mol Cell Res 1867:118664

115. Buccarello L, Sclip A, Sacchi M et al (2017) The c-jun N-terminal kinase plays a key role in ocular degenerative changes in a mouse model of Alzheimer disease suggesting a correlation between ocular and brain pathologies. Oncotarget 8(47):83038-83051

116. Xiao ZY, Wang B, Fu W et al (2018) The hippocampus is a critical site mediating antidepressant-like activity of apelin-13 in rats. Neuroscience 375:1-9

117. Dinkova-Kostova AT, Abramov AY (2015) The emerging role of Nrf2 in mitochondrial function. Free Radic Biol Med 88:179-188

118. Ding R, Wu W, Sun Z et al (2020) AMP-activated protein kinase: an attractive therapeutic target for ischemia-reperfusion injury. Eur J Pharmacol 888:173484

119. Li E, Deng H, Wang B et al (2016) Apelin-13 exerts antidepressant-like and recognition memory improving activities in stressed rats. Eur Neuropsychopharmacol 26:420-430

120. Yamazaki Y, Kanekiyo T (2017) Blood-brain barrier dysfunction and the pathogenesis of Alzheimer's disease. Int J Mol Sci 18(9): 1965

121. Azizi Y, Faghihi M, Imani A et al (2015) Post-infarct treatment with [Pyr(1)]apelin-13 improves myocardial function by increasing neovascularization and overexpression of angiogenic growth factors in rats. Eur J Pharmacol 761:101-108

122. Vagnucci AH Jr, Li WW (2003) Alzheimer's disease and angiogenesis. Lancet 361:605-608

123. Kim S, Kim S et al (2020) Apelin-13 inhibits methylglyoxalinduced unfolded protein responses and endothelial dysfunction via regulating AMPK pathway. Int J Mol Sci 21(11):4069

124. Hall C, Ehrlich L, Venter J et al (2017) Inhibition of the apelin/ apelin receptor axis decreases cholangiocarcinoma growth. Cancer Lett 386:179-188

125. Chu H, Yang X, Huang C et al (2017) Apelin-13 protects against ischemic blood-brain barrier damage through the effects of aquaporin-4. Cerebrovasc Dis 44:10-25

126. Samura M, Morikage N, Suehiro K et al (2013) Combinatorial treatment with apelin-13 enhances the therapeutic efficacy of a preconditioned cell-based therapy for peripheral ischemia. Sci Rep 6:19379

127. Toledo JB, Arnold SE, Raible K et al (2013) Contribution of cerebrovascular disease in autopsy confirmed neurodegenerative disease cases in the National Alzheimer's Coordinating Centre. Brain 136:2697-2706

128. Greenberg SM, Bacskai BJ, Hernandez-Guillamon M et al (2020) Cerebral amyloid angiopathy and Alzheimer disease-one peptide, two pathways. Nat Rev Neurol 16(1):30-42

129. Klohs J (2019) An integrated view on vascular dysfunction in Alzheimer's disease. Neurodegener Dis 19:109-127. https://doi. org/10.1159/000505625 
130. Weller RO, Subash M, Preston SD et al (2008) Perivascular drainage of amyloid-beta peptides from the brain and its failure in cerebral amyloid angiopathy and Alzheimer's disease. Brain Pathol 18:253-266

131. Tanaka M, Saito S, Inoue T et al (2020) Potential therapeutic approaches for cerebral amyloid angiopathy and Alzheimer's disease. Int J Mol Sci 21(6):1992

132. Salminen A, Kauppinen A, Kaarniranta K (2017) Hypoxia/ ischemia activate processing of amyloid precursor protein: impact of vascular dysfunction in the pathogenesis of Alzheimer's disease. J Neurochem 140:536-549

133. Katusic ZS, Austin SA (2014) Endothelial nitric oxide: protector of a healthy mind. Eur Heart J 35:888-894

134. Serrano-Pozo A, Das S, Hyman BT (2021) APOE and Alzheimer's disease: advances in genetics, pathophysiology, and therapeutic approaches. Lancet Neurol 20:68-80

135. Safieh M, Korczyn AD, Michaelson DM (2019) ApoE4: an emerging therapeutic target for Alzheimer's disease. BMC Med 17(1):64

136. Scheltens P, Blennow K, Breteler MM et al (2016) Alzheimer's disease. Lancet 388(10043):505-517. https://doi.org/10.1016/ S0140-6736(15)01124-1

137. Rebeck GW, Reiter JS, Strickland DK et al (1993) Apolipoprotein $\mathrm{E}$ in sporadic Alzheimer's disease: allelic variation and receptor interactions. Neuron 11:575-580

138. Li Z, Shue F, Zhao N et al (2020) APOE2: protective mechanism and therapeutic implications for Alzheimer's disease. Mol Neurodegener 15:63

139. Castellano JM, Kim J, Stewart FR et al (2011) Human apoE isoforms differentially regulate brain amyloid-beta peptide clearance. Sci Transl Med 3:89
140. Boehm-Cagan A, Bar R, Liraz O et al (2016) ABCA1 agonist reverses the ApoE4-driven cognitive and brain pathologies. $\mathrm{J}$ Alzheimers Dis 54:1219-1233

141. Liu XY, Lu Q, Ouyang XP et al (2013) Apelin-13 increases expression of ATP-binding cassette transporter A1 via activating protein kinase $\mathrm{C}$ alpha signaling in THP-1 macrophage-derived foam cells. Atherosclerosis 22:398-407

142. Wiesmann M, Roelofs M, van der Lugt R et al (2017) Angiotensin II, hypertension and angiotensin II receptor antagonism: roles in the behavioural and brain pathology of a mouse model of Alzheimer's disease. J Cereb Blood Flow Metab 37(7):2396-2413

143. Zhu D, Shi J, Zhang Y et al (2011) Central angiotensin II stimulation promotes $\beta$ amyloid production in Sprague Dawley rats. PLoS ONE 6(1):e16037

144. Tian M, Zhu D, Xie W et al (2012) Central angiotensin IIinduced Alzheimer-like tau phosphorylation in normal rat brains. FEBS Lett 586(20):3737-3745

145. Siddiquee K, Hampton J, McAnally D et al (2013) The apelin receptor inhibits the Ang II type 1 receptor via allosteric transinhibition. Br J Pharmacol 168:1104-1117

146. Tong Q, Wu L, Jiang T et al (2016) Inhibition of endoplasmic reticulum stress-activated IRE1alpha-TRAF2-caspase-12 apoptotic pathway is involved in the neuroprotective effects of telmisartan in the rotenone rat model of Parkinson's disease. Eur J Pharmacol 776:106-115

147. Zhang J, Lin X, Xu J et al (2019) Apelin-13 reduces oxidative stress induced by uric acid via downregulation of renin-angiotensin system in adipose tissue. Toxicol Lett 305:51-57

Publisher's Note Springer Nature remains neutral with regard to jurisdictional claims in published maps and institutional affiliations. 